\title{
Tratamento do Diabetes Mellitus do Tipo 2: Novas Opções
}

\section{RESUMO}

O diabetes mellitus do tipo 2 (DM2) resulta de defeitos na secreção e ação da insulina. Ele está freqüentemente associado à resistência à insulina, obesidade andróide, dislipidemia e hipertensão arterial, constituindo a síndrome metabólica. O tratamento atual visa diminuir a resistência à insulina e melhorar a função da célula beta pancreática com dieta, exercícios, hipoglicemiantes orais, anti-hiperglicemiantes e/ou drogas anti-obesidade. Novas drogas no tratamento do DM estão surgindo, tornando possiveis múltiplas opçōes terapêuticas. Este artigo apresenta uma revisão sobre o assunto. (Arq Bras Endocrinol Metab 2000; 44/6: 509-18)

Unitermos: Diabetes tipo 2; Diabetes mellitus não insulino-dependente; Hipoglicemiantes orais; Anti-hiperglicemiantes; Tratamento.

\begin{abstract}
Type 2 diabetes mellitus results from defects in both insulin action and insulin secretion. It is frequently associated with hyperinsulinemia, central obesity, dyslipidemia and arterial hypertension, components of the so-called metabolic syndrome. The main targets of the treatment are to decrease insulin resistance and improve beta cell function with diet, exercise, oral hypoglycemic and anti-hyperglycemic agents and/or anti-obesity drugs. New drugs for the treatment of diabetes mellitus make possible multiples therapeutics options. This article reviews this subject. (Arq Bras Endocrinol Metab 2000; 44/6: 509-18)
\end{abstract}

Keywords: Type 2 diabetes; Non-insulin dependent diabetes mellitus: Oral hypoglycemic drugs; Anti-hyperglycemic agents; Treatment.

O Diabetes mellitus do TiPO 2 (DM2) ou não insulino-dependente e a intolerância à glicose têm se tornado um dos distúrbios mais comuns em clinica médica e estão freqüentemente associados à síndrome metabólica, que se caracteriza por resistência à insulina, obesidade andróide ou central, dislipidemia e hipertensão arterial $(1,2)$. A síndrome metabólica é responsável pela maior morbi-mortalidade por doenças cardiovasculares tanto em obesos quanto em diabéticos tipo 2 .

O tratamento atual do DM2 visa manter o controle glicêmico adequado, seja com dieta hipocalórica, aumento da prática de exercícios físicos ou uso de medicações. Existem no momento diversas opções terapêuticas, que podem ser utilizadas isoladamente ou em associaçōes: sensibilizadores da ação de insulina (metformina, tiazolidinedionas), anti-hiperglicemiantes (acarbosc), secretagogos (sulfoniluréias, repaglinida, nateglinida), drogas anti-obesidade e/ou insulina. O Diabetes Control and Complications Trial (DCCT) (3), um estudo prospectivo multicêntrico sobre as diversas formas de tratamento com insulina no DMl e, mais recentemente,

\section{perspectivas}

\author{
Leila Maria Batista Araújo \\ Maria M. dos Santos Britto \\ Thomaz R. Porto da Cruz
}

\author{
Disciplina de Endocrinologia \\ da Universidade Federal da Babia, \\ Salvador, $B A$.
}


o United Kingdom Prospective Diabetes Study (UKPDS) (4), em DM2 tratados com dieta, insulina $\mathrm{e} /$ ou hipoglicemiantes orais, mostraram a fundamental importância do controle glicêmico na prevenção ou redução das complicações micro e macrovasculares. O UKPDS mostrou que o desenvolvimento de complicações microvasculares no DM2 foi reduzido, quer tratados com sulfoniluréia, metformina ou insulina, mas apenas os pacientes tratados com a metformina apresentaram diminuição significativa das complicaçōes macrovasculares (4). O UKPDS também mostrou que ocorre uma piora progressiva da função da célula beta pancreática, independente do tipo de tratamento, e que após 9 anos de tratamento apenas $25 \%$ dos diabéticos continuam a responder à monoterapia, sendo necessário associações de múltiplos recursos para mclhor controle glicêmico.

É essencial conscientizar o diabético da sua participação no controle glicêmico, com determinação de glicemia capilar, como proceder em situaçōes de hiper ou hipoglicemia, nas infeç̧òes e na gravidez. Isto melhora o controle do DM e diminui a frequiência de internações hospitalares.

\section{DIETA}

A alimentação do diabético deve ser individualizada de acordo com as necessidades calóricas diárias, atividade física e hábitos alimentares. No indivíduo não diabético, calcula-se o gasto calórico como sendo de 30 a 40 calorias $/ \mathrm{kg} /$ dia. No DM2 obeso, o que ocorre em 85 a $90 \%$ dos casos, dever-se-á diminuir o valor calórico diário $\mathrm{cm} 15$ a $30 \%$ ou mais. Isto por si só já reduziria três dos fatores de risco para doença cardiovascular, que são: a obesidade, a dislipidemia (presente em cerca de um terço dos diabéticos) e a hipertensão arterial. Uma dieta hipocalórica por si só melhora a sensibilidade à insulina e reduz a hiperglicemia, independente da perda de peso.

Quanto ao teor dos carboidratos da dieta, recomenda-se de 50 a $60 \%$ do total de calorias. Deve ser incentivado o consumo de alimentos ricos em fibras, 20 a $35 \mathrm{~g}$ por dia, as quais estão presentes nos legumes, raízes e tubérculos (5). As fibras agem retardando o esvaziamento gástrico, diminuindo a absorção intestinal de glicose e os níveis de LDL-colesterol, além de facilitar o trânsito intestinal. O teor de proteínas deve ser entre 10 e $20 \%$ do total das calorias, uma vez que a dieta rica em proteínas e a hiperglicemia podem aumentar a taxa de filtração glomerular e ainda não se sabe se interferem na progressão da nefropatia diabética. Para pacientes com nefropatia, recomenda- se 0,6 a $0,8 \mathrm{~g} / \mathrm{kg}$ de peso de proteína ou menos. As proteínas de origem vegetal têm a vantagem de conter muito menor teor de gordura do que a animal, as quais geralmente são insaturadas. $\mathrm{O}$ teor de gorduras deve ser menor do que $30 \%$ do total de calorias da dieta, evitando-se as gorduras saturadas, de procedência animal, além das exceções vegetais, a do côco e a do dendê. A ingestão de colesterol deve ser menor que $300 \mathrm{mg}$ por dia. Recomenda-se $10 \%$ de gordura poliinsaturada (óleo de soja, milho, arroz) e 10\% de gordura mono-insaturada (óleo de oliva, canola, etc) e $10 \%$ ou menos de gordura saturada (5).

Se existe hipercolesterolemia associada, deve-se limitar a ingesta diária de gorduras saturadas para menos do que $7 \%$ do total e o colesterol menor do que 200 $\mathrm{mg} / \mathrm{dl}$. Se existe hipertrigliceridemia, além de se incentivar a perda de peso, a atividade física e a restrição de álcool, recomenda-se que o teor de gorduras seja de $20 \%$ ou menos do total de calorias ( $10 \%$ saturada e poli-insaturada e $10 \%$ em mono-insaturada) e a reduçào da ingesta de carboidratos de absorção rápida.

\section{EXERCICIOS FÍSICOS}

O exercício melhora a sensibilidade à insulina, diminui a hiperinsulinemia, aumenta a captação muscular de glicose, melhora o perfil lipídico e a hipertensão arterial, além da sensação de bem-estar físico e psíquico decorrente; também pode contribuir para a perda de peso. Deve ser feita cuidadosa avaliação quanto à presença de complicações macro e microvasculares no DM, para definir os tipos de exercícios mais apropriados, se neuropatia, nefropatia e/ou retinopatia estiverem presentes (6).

Deve-se evitar a aplicação de insulina nos locais adjacentes aos principais músculos envolvidos, para evitar absorção mais rápida da insulina. Recomenda-se caminhadas pelo menos 3 a 4 vezes por semana durante 30 a 40 minutos. Os relatos na literatura sugerem que a prática de exercício físico induz uma diminuição dos níveis de triglicérides de 11 a 16\%, do colesterol de 3 a $10 \%$ e aumento do HDL-colesterol em 3\% (7).

No indivíduo com glicemia abaixo de 100 $\mathrm{mg} / \mathrm{dl}$, os exercícios físicos podem provocar hipoglicemia e, a depender do tipo e duração do exercício, recomenda-se um pequeno lanche rico em carboidrato antes de iniciá-lo. Já o diabético com níveis glicêmicos maiores do que $250 \mathrm{mg} / \mathrm{dl}$ com cetose ou maiores do que $300 \mathrm{mg} / \mathrm{dl}$, deve evitá-los.

Um estudo prospectivo em 110.660 indivíduos seguidos por 6 anos mostrou que a prática diária de exerć́cios físicos diminuiu a incidência de DM em $46 \%$ (8). 


\section{HIPOGLICEMIANTES ORAIS, ANTI- HIPERGLICEMIANTES E SENSIBILIZADORES DA AÇÃO DE INSULINA}

$\mathrm{O}$ uso destas medicações está indicado no DM2, quando com a dieta $\mathrm{e} o$ aumento da atividade física não forem capazes de obter um bom controle, ou seja, glicemias de jejum e pós prandial e hemoglobina glicosilada próximos aos níveis normais $(7,8)$.

No DM2 obeso, a prioridade é a perda de peso. Caso não se consiga após 4 a 6 semanas controle glicê- mico adequado, podem ser indicadas inicialmente drogas que sensibilizam a ação de insulina (biguanida e tiazolidinediona), associadas ou não a drogas antiobesidade. Caso ainda não se consiga um controle glicêmico satisfatório, podem ser associadas drogas que diminuam a absorção intestinal de glicose (acarbose ou miglitol), ou que aumentem a secreção de insulina (sulfoniluréia, repaglinida ou netaglinida) (tabela 1 ).

No diabético tipo 2 de peso normal ou com excesso de peso (índice de massa corpórea $<30 \mathrm{~kg} / \mathrm{m}^{2}$ ),

Tabela 1. Hipoglicemiantes orais, anti-hiperglicemiantes e sensibilizadores da ação de insulina disponiveis: apresentação, duração de ação e dose máxima.

\begin{tabular}{|c|c|c|c|c|}
\hline Droga & $\begin{array}{l}\text { Nome } \\
\text { Comercial }\end{array}$ & Apresentação & $\begin{array}{l}\text { Duração } \\
\text { de ação }\end{array}$ & $\begin{array}{l}\text { Dose } \\
\text { Máxima }\end{array}$ \\
\hline
\end{tabular}

\section{Biguanidas}

$\begin{array}{lllll}\text { Metformina } & \begin{array}{l}\text { Dimefor } \\ \text { Glifage } \\ \text { Glucoformin } \\ \text { Metformina }\end{array} & \begin{array}{l}\text { Comp. } 850 \mathrm{mg} \\ \text { Comp. } 500 \text { e } 850 \mathrm{mg}\end{array} & 9-12 \mathrm{~h} & 2500 \mathrm{mg} \\ \text { Fenformina } & \text { Debei } & \text { Comp. } 50 \mathrm{mg} & 4-6 \mathrm{~h} & 150 \mathrm{mg}\end{array}$

\section{Inibidores da Alfa-Glucosidase}

Acarbose

Miglitol

\section{Tiazolidinedionas}

\begin{tabular}{ll}
$\begin{array}{l}\text { Rosiglitazona } \\
\text { Pioglitazona }\end{array}$ & $\begin{array}{l}\text { Avandia } \\
\text { Actos }\end{array}$ \\
4. Sulfoniluréias & \\
Clorpropamida & $\begin{array}{l}\text { Clorpropamida } \\
\text { Diabinese }\end{array}$ \\
Glibenclamida & $\begin{array}{l}\text { Aglucil, Apex } \\
\text { Daonil } \\
\text { Euglucon } \\
\text { Glibenclamida } \\
\text { Lisaglucon }\end{array}$ \\
\hline
\end{tabular}

$\begin{array}{lll}\text { Gliclazida } & \text { Diamicron } & \text { Comp. } 80 \mathrm{mg} \\ \text { Glimepirida } & \text { Amaryl } & \text { Comp. 1. } 2 \text { e } 4 \mathrm{mg} \\ \text { Glimepil } & \\ \text { Glipizida } & \begin{array}{l}\text { Minidiab } \\ \text { Glipizida }\end{array} & \text { Comp. } 5 \mathrm{mg}\end{array}$

Glucobay

Comp. 50 e $100 \mathrm{mg}$

Comp. 25, 50 e $100 \mathrm{mg}$

$4 h$

$2-4 h$

$300 \mathrm{mg}$

Glyset

Comp. 4 e $8 \mathrm{mg}$

Comp. 15, 30 e $45 \mathrm{mg}$

$12-24 h$

$16-24 h$

$8 \mathrm{mg}$

$45 \mathrm{mg}$

\section{Nateglinida}

\section{Metiglinida}

Repaglinida

Novonorm

Prandin

Nateglinida

Starlix

Comp. 60,120 e $180 \mathrm{mg}$

$1,6 \mathrm{~h}$

$720 \mathrm{mg}$

$6-12 h$

$240 \mathrm{mg}$

$6-24 h$

$8 \mathrm{mg}$

$6-12 h$

$20 \mathrm{mg}$ 
pode ser tentado inicialmente sulfoniluréia, repaglinida ou nateglinida e, se após 2 a 4 semanas não obtiver um controle glicêmico adequado, podem ser associados biguanida, tiazolidinediona ou um inibidor da absorção intestinal de glicose.

\section{Biguanidas}

Em relação às biguanidas, temos disponíveis a metformina e a fenformina, sendo a primeira mais utilizada, pela menor freqüência de efeitos colaterais (8-14).

A metformina melhora a ação da insulina no fígado, diminuindo a produção hepática da glicose em 10 a $30 \%$ e, no músculo, aumentando a captação de glicose em 15 a $40 \%$ c estimulando a glicogênese. No adipócito, a metformina inibe a lipólise e a disponibilidade de ácidos graxos livres (FFA). Tem sido descrito que esta droga aumenta o númcro $c$ mclhora a afinidade dos receptores de insulina, tanto no adipócito, quanto no músculo. A nível celular foi demonstrado que a metformina aumenta a atividade da tirosinaquinase do receptor da insulina, estimulando a translocação do GLUT4 e a atividade da glicogênio-sintetase (10). A secreção de insulina aos estímulos pode permanecer inalterada ou diminuir. Ocorre também melhora do perfil lipídico, com diminuiçăo de 20 a $25 \%$ nos níveis de triglicérides e de até $10 \%$ do LDL-colesterol, e aumento de até 17\% dos níveis de HDL-colesterol, com diminuição de 20 a $30 \%$ do fator inibidor de ativação do plasminogênio (PAI-l). Seu efeito anorético auxilia na perda de peso. A outra vantagem é não provocar hipoglicemia, por não estimular a secreção de insulina.

No DM2, o uso isolado de metformina diminui a glicemia cerca de $25 \%$, ou 60 a $70 \mathrm{mg} / \mathrm{dl} \mathrm{e}$ a hemoglobina glicosilada em 1,5 a $2 \%$ (9). Foi demonstrado, também, que a eficácia do controle glicêmico pela metformina é similar à da sulfonilurćia, além de estar associada à redução ponderal, tão importante no DM2 (11). O estudo multicêntrico do UKPDS em diabéticos acompanhados por cerca de 11 anos mostrou que o controle intensivo da glicemia pelo uso de metformina diminuiu significativamente os riscos de doença cardiovascular e a mortalidade relacionada ao DM, associado com menor ganho de peso e sem a inconveniência das hipoglicemias induzidas pelo tratamento com insulina ou sulfoniluréias (13). Neste estudo, contudo, o número de pacientes diabéticos tratados com a metformina foi menor que $10 \%$ do total de pacientes observados.

A metformina é apresentada em comprimidos de 500 e $850 \mathrm{mg}$ e a dose máxima a ser utilizada é de $2,5 \mathrm{~g} / \mathrm{dia}$, embora na literatura haja relato de uso de até
$3 \mathrm{~g}(9)$, sempre administrada após as refeiçōes para minimizar os efeitos gastrointestinais. A metformina é absorvida no intestino e excretada pelos rins, quase não sendo metabolizada pelo fígado, enquanto $30 \%$ da fenformina têm metabolização hepática. A metformina também difere da fenformina por ter baixa afinidade pelas membranas mitocondriais e por não interferir na fosforilização oxidativa. Está indicada como monoterapia nos diabéticos obesos ou mesmo intolerantes à glicose. Cerca de 5 a $10 \%$ dos pacientes por ano deixam de ter uma resposta adequada. Quando não se consegue um controle satisfatório, pode ser utilizada em associação com sulfoniluréia (14), acarbose (15), tiazolidinediona (16), repaglinida (17) e/ou insulina $(8,12)$.

Os efeitos colaterais mais freqüentes são diarréia (15\%), gosto metálico e náuseas, os quais por vezes diminuem com a continuidade do uso da medicação. Diminuição da absorção da vitamina Bl2 tem sido descrita. A metformina tem efeito sinérgico com a cimetidina (9). Acidose lática com o uso de metformina é rara $(0,03$ a $0,4 / 1000 / \mathrm{ano})$ e tem sido mencionada na maioria das vezes em indivíduos nos quais esta droga estaria contra-indicada, tais como doença crônica do fígado com elevação de transaminases 2 a 3 vezes os valores normais, insuficiência cardíaca, respiratória ou renal (clearance $<70 \mathrm{ml} / \mathrm{min}$ ou creatinina sérica $\geq 1,5 \mathrm{mg} / \mathrm{dl}$ ). Não é aconselhável o seu uso em indivíduos com mais de 80 anos, gestantes, lactentes ou em alcoólatras. Em paciente com proteinúria que for submetido a exame radiológico contendo iodo, é prudente suspender a medicação alguns dias antes $\mathrm{e}$ receber uma hidratação adequada.

\section{Inibidores da Alfa-glicosidase}

Os inibidores competitivos da alfa-glicosidase, como a acarbose, o miglitol e a voglibose agem como antagonistas enzimáticos da amilase e sucrase e diminuem a absorção intestinal da glicose $(18,19)$. Estas medicações não interferem na secreção de insulina, e diminuem a glicemia de jejum e a hiperglicemia pós-prandial. Observou-se diminuição dos níveis de triglicérides com o uso de miglitol.

Os efeitos colaterais mais freqüentes são flatulência ( 20 a $55 \%$ ), diarréia ( 3 a $14 \%$ ), dores abdominais ( 8 a $21 \%$ ) e elevação das transaminases, que têm relação com a dose utilizada e são atenuados com a continuidade. A acarbose é disponível $\mathrm{cm}$ comprimidos de 50 e $100 \mathrm{mg}$, devendo ser administrada com a primeira porção das principais refeições. A dose diária máxima é de $300 \mathrm{mg}$, cmbora seja referido o uso de até $600 \mathrm{mg}$. Podem ser associadas a outro antidiabético oral ou insulina $(15,20)$. Deve ser ressaltado que, em caso de 
hipoglicemia, recomenda-se à administraçào de glicose oral, uma vez que a sacarose não é absorvida.

As contra-indicações para o uso desta droga são as doenças intestinais inflamatórias, gravidez e lactaçào, insuficiência hepática ou renal grave.

\section{Tiazolidinedionas}

Nos Estados Unidos da América e em alguns países da Europa, outras classes de hipoglicemiantes derivadas da tiazolidinediona $(23,24)$ estão disponívcis comercialmente há algum tempo, tendo recentemente sido retirada do mercado a troglitazona, devido à toxicidade hepática.

As tiazolidinedionas de segunda geração, rosiglitazona e pioglitazona, agem aumentando e sensibilizando a ação da insulina no fígado, músculos e adipócitos, diminuindo a resistência periférica. Elas ativam os receptores nucleares intracelulares (PPAR-g, peroxisome proliferator activated receptor) que regulam a expressão de genes que afetam o metabolismo glicídico e lipídico, responsáveis pela captação de glicose mediada por insulina nos tecidos periféricos e pela diferenciação de pré-adipócitos em adipócitos, entre outros efeitos. As tiazolidinedionas aumentam a expressão dos transportadores de glicose (GLUT4), da lipoproteína lipase e reduzem a expressão da leptina e do fator de necrose tumoral (TNF-alfa).

As tiazolidinedionas inibem a oxidação de cadeias longas de ácidos graxos no fígado, diminuindo a gliconeogênese e a disponibilidade de ácidos graxos livres. Embora induzam a diminuição de $\mathbf{1 5}$ a $20 \%$ dos níveis de triglicérides c aumento de 5 a $10 \%$ do HDLcolesterol, os níveis de colesterol total e do LDL-colesterol podem não se alterar ou aumentar de 10 a $15 \%$. É possível que as tiazolidinedionas diminuam a progressão da aterosclerose, pois em ratos submetidos à injúria arterial a troglitazona inibiu o crescimento das células da musculatura lisa vascular e da hiperplasia da íntima. Em diabéticos tratados com troglitazona foram observadas diminuições da adesividade plaquetária, do fator inibidor de ativação do plasminogênio (PAI-1) e dos níveis pressóricos. Estes múltiplos efeitos fortalecem a sua indicação no tratamento da síndrome metabólica.

As tiazolidinedionas diminuem os níveis glicêmicos em torno de $20 \%$, mas não aumentam a secreção de insulina. Quando comparada à metformina, observou-se que a troglitazona têm maior efeito potencializador da ação periférica da insulina e menor efeito em diminuir a produção hepática de glicose (16). A associaçào de tiazolidinediona com metformina é interessante, pois tem efeitos aditivos. A pioglita- zona pode alterar os níveis de drogas de metabolização pelas enzimas $\mathrm{P}_{450}$, diminuindo cerca de $30 \%$ os níveis de etinil-estradiol e noretindrona, devendo ser aumentada a dose de contraceptivos naquelas diabéticas que não desejam engravidar. Pode ser utilizada como monoterapia ou $\mathrm{em}$ associação com a metformina, com a qual tem efeito anti-hiperglicemiante potencializado, ou com a sulfoniluréia, meglitinida, ou mesmo insulina, especialmente naqueles diabéticos com síndrome metabólica. Entretanto, devido à possibilidade de complicaçōes hepáticas, inclusive relato de casos fatais com o uso de troglitazona, recomenda-se cautela com a sua indicação, como também em cardiopatas, pela possibilidade de edema.

A rosiglitazona é mais potente e tem menor toxicidade hepática do que a troglitazona (24), não induzindo metabolismo pelo citocromo $\mathrm{P}_{450}(\mathrm{CYP})$ $3 \mathrm{~A} 4$, e não havendo, assim, interação com contraceptivos orais, digoxina, ranitidina e nifedipina, entre outras. A dose indicada da rosiglitazona varia de 4 a $8 \mathrm{mg}$, e da pioglitazona de 15 a $45 \mathrm{mg}$, podendo ambas serem administradas uma vez ao dia. A farmacocinética de ambas não é alterada pela insuficiência renal leve a moderada, não requerendo alteração da dose.

Os efeitos colaterais ocorrem em menos de $5 \%$ dos pacientes: infecções do trato respiratório supcrior, cefaléia, elevação de transaminases, edema, aumento de peso e anemia. Hipoglicemia pode ocorrer quando o seu uso for concomitante com secretagogos ou insulina. Contraindica-sc o seu uso $\mathrm{cm}$ crianças, gravidez, hepatopatias ou em indivíduos com elevação dos níveis de transaminases de 2 a 3 vezes os valores de referência.

A pioglitazona também tem menor toxicidade hepática que a troglitazona, mecanismo de ação e efeitos colaterais similares a rosiglitazona, mas tem interação com algumas medicações, entre as quais etinil-estradiol e noretindrona, diminuindo sua concentração em $30 \%$, com perda do efeito contraceptivo.

\section{Sulfoniluréias}

As sulfoniluréias ( $\mathrm{SU}$ ) de primeira geraçào, como a clorpropamida, têm mcia vida mais longa (36 a 60h), devido à formação de metabólitos com excreção mais lenta. Já as de $2^{a}$ geração, como a glibenclamida, gliclazida e glipizida e as de $3^{a}$ geraçào, como a glimepiri$\mathrm{da}$, têm meia vida mais curta, de 8 a $16 \mathrm{~h}(7,8)$. A escolha do tipo da SU depende da idade do paciente, da tolerabilidade e da resposta à medicação. A glicazida diminui a oxidação de LDL-lipoproteínas e a adesão de monócitos no endotélio. Existem receptores 
de $S U$ específicos na célula beta pancreática e em outros tecidos. A glimepirida, a mais recente das $\mathrm{SU}$, tem maior ligação com as proteínas do pâncreas (canais de potássio ATP-sensiveis, $\mathrm{K}_{\mathrm{ATP}}$ ) e menor ligação com as do miocárdio, em relação às outras SU. A glimepirida tem associação e dissociação mais rápida com os sítios de ligação dos receptores de SU, levando a uma liberaçào mais rápida e duração da ação mais curta da insulina.

O mecanismo de ação das SU não está bem esclarecido. No DM2, uma das possíveis causa da perda da sensibilidade da célula beta pancreática ao estímulo da hiperglicemia, está na regulação dos canais de $\mathrm{K}_{\mathrm{ATP}}$. A nível celular, as $\mathrm{SU}$ agem inibindo os canais de $\mathrm{K}_{\mathrm{ATP}}$, despolarizando a célula beta pancreática $\mathrm{c}$ estimulando o influxo de $\mathrm{Ca}^{2+} \mathrm{e}$ a secreção de insulina.

A curto prazo, as SU aumentam a secreção de insulina, mas a longo prazo (mais de 6 meses), a secreção de insulina pode estar igual ou até diminuída em relação aos níveis iniciais, mas o efeito hipoglicemiante persiste e possivelmente está relacionado aos efeitos extra-pancreáticos (25). Alguns estudos sugerem que elas aumentem o número de receptores de insulina e/ou tenham efeito pós-receptor, facilitando as ações da insulina. As $S U$ não restauram a primeira fase de secreção de insulina ao estímulo da glicose venosa, mas exageram a segunda fase, daí a importância de usar SU de ação mais curta.

A principal indicação de SU é em DM2, com peso normal ou magro, nos quais os nívcis de insulina estão normais ou diminuídos, nos quais não se consiga um controle satisfatório com dieta e exercícios, na ausência de infecção ou cetose.

Recomenda-se iniciar com um comprimido, em jejum, observar a resposta por 2-3 semanas e, se necessário, aumentar a dose. A dose máxima diária varia para cada droga: clorpropamida - $500 \mathrm{mg}$, glibenclamida - $20 \mathrm{mg}$, gliclazida - $320 \mathrm{mg}$, glipizida $20 \mathrm{mg}$ e glimepirida - $8 \mathrm{mg}$. Os comprimidos devem ser administrados em jejum ou antes da refeição. Quando o controle glicêmico não for satisfatório, as SU podem ser associadas a metformina (14) e/ou à acarbose (20), à troglitazona $(22,23)$ ou à insulina $(26)$. Foi descrito ainda uso de associação de glibenclamida com mais duas outras drogas hipoglicemiantes: metformina e acarbose (27).

Os efeitos colaterais são observados em 3 a $5 \%$ dos pacientes: hipoglicemia, alterações hematológicas (leucopenia, agranulocitose, trombocitopenia e anemia hemolítica) e gastro-intestinais (náusea, vômito, mais raramente icterícia colestática) e reações alérgicas. Pacientes $\mathrm{cm}$ uso de clorpropamida podem apresentar sensação de calor e vermelhidāo após uso concomitante de bebidas alcoólicas (efeito antabuse).

Outro cfeito indesejável é a tendência ao aumento de peso, relacionado ao aumento dos níveis insulinêmicos. A hipoglicemia é mais comum com o uso de SU de ação prolongada, que têm ligação iônica com as proteínas plasmáticas e pode ser potencializada pelo uso concomitante de salicilatos, sulfa, fenilbutazona, isoniazida, álcool e fibratos, entre outros. Pode haver interação da glimepirida com o miconazol e potencial interação com drogas de metabolização pelo citocromo $\mathrm{P}_{450}$ II C9. A cardiotoxidade das SU ainda nào está definida, embora alguns estudos sugiram que pode agravar a hipoxemia do miocárdio no caso de doença coronariana.

Cerca de 30\% dos diabéticos pode ter falha primária à $\mathrm{SU}$ e os fatores preditivos são: idade menor que 40 anos, duração do DM maior que 5 anos e peso normal ou baixo. Falha secundária é também comum após anos de uso de SU. O UKPDS mostrou que ocorre uma progressiva perda da função da célula beta pancreática independente do tipo de tratamento. Após 6 anos de uso de $\mathrm{SU}$ apenas $52 \%$ dos pacientes continuam a ter uma função da célula beta pancreática adequada (4).

A metabolização das SU é hepática (cerca de $60 \%)$ e a excreção é renal. As contra-indicações para seu uso são a presença de insuficiência hepática ou renal, gravidez, presença de infecção, cetose e no DM secundário a pancreatite. Nos indivíduos idosos (mais de 60 anos) e nefropatas, evita-se o uso de SU de primeira geração.

\section{Outros Secretagogos de Insulina: Repaglinida e Nateglinida}

A meglitinida é um grupo de substâncias derivadas do ácido benzóico (dentre as quais a repaglinida), que aumenta a secreção de insulina, com ação semelhante à da $\mathrm{SU}$, bloqueando os canais de $\mathrm{K}_{\mathrm{ATP}}$ da célula beta pancreática, mas atuando na subunidade reguladora desses canais em sítios de ligação diferente das SU.

Nos indivíduos com DM2 ocorre perda da secreção rápida ou precoce da insulina, responsável pela supressão da produção hepática da glicose, limitando a hiperglicemia pós-prandial e o hiperinsulinismo tardio, e melhorando a tolerância a glicose, com redução do risco de hipoglicemia interprandial. As meglitinidas restauram esta secreção e, portanto, diminuem a hiperglicemia pós-prandial, à qual tem sido atribuído papel importante na patogênese das complicações do DM.

A repaglinida é rapidamente absorvida e sua ação começa dentro de 30 minutos, facilitando a fase 
de secreção rápida de insulina, devendo ser administrada imediatamente antes ou com a refeição, para evitar hipoglicemia. Um estudo comparativo entre gliburida e repaglinida mostrou que o efeito hipoglicemiante é similar (28). A repaglinida tem metabolização hepática, sendo $90 \%$ excretada pela bile; assim, deve ser evitada em pacientes com doença hepática. Seu metabolismo é alterado por drogas que induzem o citocromo $\mathrm{P}_{450} 3 \mathrm{~A} 4$, como: cetoconazol, eritromicina, etc. Os efeitos colatcrais mais freqüentes são hipoglicemia, ganho de peso, sintomas de rinite e sinusite, cefaléia, diarréia e artralgias. Pode ser utilizada como monoterapia ou ser associada à metformina $(17,29)$ ou às glitazonas.

A nateglinida é derivada de um aminoácido, a D-fenilalanina, e age restaurando a fase de liberação rápida da insulina e evitando os picos hiperglicêmicos pós-prandiais. A nateglinida tem início de ação rápido e tem o pico da insulina um pouco mais rápido do que a repaglinida $(0,78$ vs $0,92 \mathrm{~h})$, com menor duração de ação. A nateglinida tem maior seletividade pelos canais de $K_{\text {ATP }}$ dependente do pâncreas do que das células cardíacas e dos vasos. Recomenda-se ser administrada imediatamente antes da alimentação e a sua absorção não sofre interferência da composição alimentar.

A dose varia de 60 a $120 \mathrm{mg}$ três vezes ao dia, a depender do controle glicêmico obtido, e pode ser associada a outras drogas. Os estudos preliminares com o uso de nateglinida em associação com metformina ou troglitazona, quando comparado a monoterapia apenas com metformina ou troglitazona, sugerem que ambas as combinações são sinérgicas com maior redução dos níveis glicêmicos do que com a monoterapia. Embora hipoglicemia sintomática seja rara $(<2 \%)$, porque sua ação na célula beta pancreática é proporcional à concentração de glicose, cla pode ocorrer quando não administrada com refeiçào ou após exercício físico extenuante.

A nateglinida não tem interação com outras medicações que competem pelos componentes do sistema $\mathrm{P}_{450}$. A nateglinida pode ser utilizada em indivíduos com $>75$ anos e com doenças concomitantes, inclusive nefropatia. Recomenda-se evitar seu uso $\mathrm{cm}$ DMl, cctoacidose, gravidez e no período de amamentaçào.

\section{DROGAS ANTI-OBESIDADE}

Uma vez que uma dieta hipocalórica com redução de peso é por si só capaz de controlar os níveis glicêmicos, em muitos pacientes diabéticos tem-se dado mais ênfase no tratamento da obesidade $(7,29)$, podendo tentar o uso de medicações anti-obesidade. Diversas drogas são disponíveis no momcnto: catecolaminérgicas (anfepramona, femproporex, mazindol), serotoninérgicas (fluoxetina, sertralina) e de ação mista catecolaminérgica e serotoninérgica (como a sibutramina), que agem no controle do apetite e na indução de saciedade.

A sibutramina (cápsulas de 10 e $15 \mathrm{mg})(30,31)$ é considerada uma droga de açào combinada de cfeitos catecolaminérgicos e serotoninérgicos, inibindo a recaptação de serotonina e de noradrenalina. Estes efeitos no sistema nervoso central levam ao aumento da saciedade e têm uma discreta ação aumentando a termogênese, por ativação dos receptores beta3 adrenérgicos no tecido gorduroso. A tolerância é muito boa, mas pode ocorrer discreta elevação da pressão e do pulso em alguns pacientes, daí um cuidado maior em cardiopatas (32).

$\mathrm{O}$ orlistat ou tetra-hidrolipstatina (cápsulas de $120 \mathrm{mg}$ ), é um inibidor da lipase intestinal que diminui em $30 \%$ a absorção de gorduras. Além da perda de peso, ocorre melhora do perfil lipídico $(31,33)$. Os estudos mais prolongados de uso desta droga, tem mostrado manutenção da perda de peso a longo prazo. $\mathrm{O}$ orlistat tem também boa tolerância $\mathrm{c}$ os efeitos colaterais mais freqüentes são diarréia com fezes gordurosas e flatulência. Um estudo multicêntrico em 391 diabéticos tratados com orlistat $(360 \mathrm{mg})$ por um ano, mostrou perda de peso duas vezes maior do que com placebo inicial após um ano, com significativa melhora do controle e do perfil lipídico e diminuição da dose de hipoglicemiante (33).

\section{INSULINA}

A indicação da insulina no tratamento do DM2 reserva-se para diabéticos sintomáticos, com hiperglicemia severa, com cetonemia ou cetonúria, mesmo recémdiagnosticados, ou para diabéticos que não respondam ao tratamento com dieta, exercício e/ou hipoglicemiante oral, anti-hiperglicemiante ou sensibilizadores da ação de insulina (35). A tabela 2 mostra os diversos tipos de insulina disponíveis.

A associação de insulina c hipoglicemiante oral parece ser benéfica em alguns casos, $(8,12,26)$. Naqueles pacientes que têm hiperglicemia pós-prandial, o uso de metformina, acarbose, repaglinida ou nateglinida, pode melhorar o perfil glicêmico, reduzir a dose de insulina e minimizar o aumento de peso $(13,35,36)$.

A insulina humana e a insulina suína associam-se freqüentemente em hexâmeros e a velocidade de dissoci- 
Tabela 2. Tipos de insulinas comercialmente disponiveis.

\begin{tabular}{|c|c|c|c|c|c|}
\hline Tipo & $\begin{array}{l}\text { Tempo } \\
\text { de Ação }\end{array}$ & Mista & Porco & Humana & Análogo \\
\hline Lispro & Ultra-rápida & & & & Humalog \\
\hline Aspart & Ultra-rápida & & & & Novorapid \\
\hline $\begin{array}{l}\text { Simples ou } \\
\text { Rápida }\end{array}$ & Rápida & Iolin-R & $\begin{array}{l}\text { Actrapid MC } \\
\text { Neosulin-R }\end{array}$ & $\begin{array}{l}\text { Biohulin-R } \\
\text { Humulin-R } \\
\text { Insuman-R } \\
\text { Novolin-R }\end{array}$ & \\
\hline $\mathrm{NPH}$ & Intermediária & Iolin-N & $\begin{array}{l}\text { Neosulin- } \mathrm{N} \\
\text { Protaphane-MC }\end{array}$ & $\begin{array}{l}\text { Biohulin-N } \\
\text { Humulin-N } \\
\text { Insuman-N } \\
\text { Novolin-N }\end{array}$ & \\
\hline $\begin{array}{l}\text { Lenta ou } \\
\text { com Zinco }\end{array}$ & Intermediária & & $\begin{array}{l}\text { Monotard MC } \\
\text { Neosulin-L }\end{array}$ & $\begin{array}{l}\text { Biohulin-L } \\
\text { Humulin-L } \\
\text { Novolin-L }\end{array}$ & \\
\hline Glargina & Intermediária & & & & Lantus \\
\hline Ultralenta & Ultra-longa & & & Biohulin-U & \\
\hline
\end{tabular}

Combinações de NPH e Regular: Biohulin (70N/30R, 80N/20R, 90N/10R), Novolin (70N/30R, 80N/20R, 90N/10N), Humulin (70N/30R, 80N/20R, 90N/10R), Insuman Comb (75N/25R e 85N/15R).

Combinaçōes de Análogos: Humalog Mix 25 (75 lispro NPL /25 lispro); Mixtard 20 ( 80 I/ 20S), Mixtard 30 (70 l/30S), Mixtard 40 $(60 \mathrm{l} / 405)$

açào em monômeros e a de absorção pelo subcutâneo são lentas. Como consequiência, a glicemia $\mathrm{l}$ a $2 \mathrm{~h}$ após a refeição se eleva muito. Quando se administra insulina regular ou simples por via subcutânea resulta numa inapropriada hiperinsulinemia ( 4 a 5 h após), a qual aumenta a chance de hipoglicemia interprandial. Os análogos de insulina de ação mais rápida do que a insulina humana diminuem este risco $(37,38)$.

Diversos tipos de análogos de insulina já foram recentemente sintetizados: a lispro, a aspart e a glargina (38). A insulina lispro (Humalog), tem ação ultra-rápida e a lispro NPL (neutral protamine lispro), tem ação intermediária (39). A lispro tem troca das posições da prolina e lisina na cadeia beta da insulina, com mais rápida dissociação de dímeros e hexâmeros em monômeros, com mais rápida absorção e ação da insulina. O pico da lispro ocorre $\mathrm{lh}$ após sua administração, com duração de efeito durante 4 h. A insulina aspart (Novorapid) tem uma substituição de prolina por ácido aspártico na posição 28 da cadeia beta da insulina e o seu pico e duração de ação é semelhante à insulina lispro. A insulina glargina (Lantus), tem ação intermediária, a qual tem modificação no peptídio terminal da cadeia beta da insulina com adição de duas moléculas de arginina e troca do aminoácido asparagina por glicina na cadeia alfa da insulina, o que the con- fere maior estabilidade e absorção contínua, mais lenta e mais prolongada, sem pico, o que a difere da insulina NPH, com menos episódios de hipoglicemia noturna. A insulina glargina não deve ser misturada com insulinas de ação rápida, mas pode ser associada à insulina lispro.

Os esquemas de administração de insulina são múltiplos. Alguns estudos sugerem que o uso de insulina de ação intermediária ao deitar associada a anti-diabético oral durante o dia é uma boa opção (40).

\section{PARÂMETROS DE BOM CONTROLE}

As determinações periódicas da hemoglobina glicosilada ou frutosamina são de grande utilidade no acompanhamento do diabético visando obtenção de um controle adequado. Considera-se bom controle quando as glicemias pré e pós-prandiais estão entre $80 \mathrm{e}$ $120 \mathrm{mg} / \mathrm{dl}$ no sangue total ou 90 e $130 \mathrm{mg} / \mathrm{dl}$ no plas$\mathrm{ma}$, antes do deitar entre $100 \mathrm{e} 140 \mathrm{mg} / \mathrm{dl}$ no sangue total ou 110 e $150 \mathrm{mg} / \mathrm{dl}$ no plasma e níveis de hemoglobina glicosilada menores que $7 \%$ ou do limite máximo dos valores normais para o método (41). Estes níveis podem ser maiores nos pacientes com história de hipoglicemias severas recorrentes, doença cardiovascular ou cérebro-vascular avançada e doença renal em 
estágio final. A frutosamina pode também ser utilizada para acompanhamento de gestantes ou para verificar o controle glicêmico nos últimos 15 a 21 dias, que é a meia vida da albumina.

É também importante o controle da dislipidemia, quando presente. Os níveis de LDL-colesterol devem ficar abaixo de $100 \mathrm{mg} / \mathrm{dl}$, de HDLcolesterol maiores do que $45 \mathrm{mg} / \mathrm{dl}$ nas mulheres e $35 \mathrm{mg} / \mathrm{dl}$ nos homens e de triglicérides abaixo de $200 \mathrm{mg} / \mathrm{dl}$, nem que seja necessário o uso de medicações hipolipemiantes (4l). Considerando que o DM é uma doença $\mathrm{em}$ que há concomitância de vários fatores de risco para doença cardiovascular, sugere-se até que os níveis de triglicérides devam ser menores do que $150 \mathrm{mg} / \mathrm{dl}$.

O UKPDS mostrou que o controle da hipertensão arterial é essencial na diminuição da morbi-mortalidade por doenças macro-vasculares em pacientes com DM2, sugerindo inclusive prioridade na normalização da tensão arterial, comparada com a busca da normoglicemia (4).

Para monitorização glicêmica domiciliar, os glicosímetros têm sido utilizados. Comercialmente existem vários tipos de glicosímetros, cada qual com sua fita reagente apropriada, que diferem entre si pelo tempo de leitura, além de outros recursos, como memória para armazenamento das leituras das glicemias, adaptação a computador, etc. $\mathrm{O}$ número de testes a serem feitos diariamente é variável. Quando o DM já está bem controlado, este número pode ser reduzido a 1 ou 2 leituras (antes do desjejum e antes ou $2 \mathrm{~h}$ após o jantar). Os testes de glicosúria com os redutores de cobre ou com inibidor da glicose-oxidase podem ser utilizados como alternativas, quando não há alteração da função renal.

A melhora do controle glicêmico do DM previne as complicaçòes agudas e crônicas, diminui a morbi-mortalidade por doenças cardiovasculares e melhora a qualidade de vida do diabético.

\section{REFERÊNCIAS}

1. Reaven $G$. Banting Lecture 1988: Role of insulin resistance in human disease. Diabetes 1998;37:1595-607.

2. Kaplan NN. The deadly quartet. Upper body obesity, glucose intolerance, hypertriglyceridemia, and hypertension. Arch Intern Med 1989;149: 1514-20.

3. The Diabetes Control and Complications Trial Research Group: the effect of intensive treatment of diabetes on the development and progression of long-term complications in insulin-dependent diabetes mellitus. JAMA 1996:276:1409-15.

4. Turner RC. The UK Prospective Diabetes Study. Diabetes Care 1998:21(suppl 3):C35-38.
5. American Diabetes Association. Nutrition recommendations and principles for people with diabetes mellitus. Diabetes Care 2000;23(suppl 1):S42-46.

6. American Diabetes Association. Diabetes mellitus and exercise. Diabetes Care 2000;23(suppl 1):S50-54.

7. Pan XR, Li GW, Hu YH, et al. Effects of diet and exercise in preventing NIDDM in people with impaired glucose tolerance. The Da Quing IGT and Diabetes Study. Diabetes Care 1997:20:537-54.

8. Hirsch IB, Riddle MC. Current therapies for diabetes. Endocr Clin North Am 1997:26:3.

9. Cusi K. DeFronzo R. Metformin: a review of its metabolic effects. Diabetes Rev 1998:6:89-131.

10. Santos RF, Normizo R, Wajchenberg BL, Reaven G, Azhar $S$. Changes in insulin receptor tyrosine kinase activity associated with metformin treatment of type 2 diabetes. Diabetes Metabol 1995:21:274-80

11. Johansen K. Efficacy of metformin in the treatment of NIDDM: meta-analysis. Diabeles Care 1999:22:33-7.

12. Hirsch IB. Metformin added to insulin therapy in poorly controlled type 2 diabetes. Diabetes Care 1999:2:854.

13. UK Prospective Diabetes Study. Effect of intensive blood-glucose control with metformin on complications in overweight patients with type 2 diabetes (UKPDS 34). UK Prospective Diabetes Study group. Lancet 1998:352:854-65.

14. DeFronzo R, Goodman AM. The multicenter metformin study group: efficacy of metformin in NIDDM patients poorly controlled on diet done or diet plus sulfonylurea. N Engl J Med 1995:333:541-9.

15. Chiasson JL, Josse RG. Hunt JA, et al. The efficacy of acarbose in the treatment of patients with non insulindependent diabetes mellitus: a multicenter controlled clinical trial. Ann Intern Med 1994;121:928-33.

16. Inzucchi SE, Maggs D. Spollett $G$, et al. Efficacy and metabolic effects of mefformin and troglitazone in type 2 diabetes mellitus. N Engl J Med 1998:338:867-72.

17. Moses R, Slobodniuk R, Donneelly T, Boyages S, et al. Effect of repaglinide addition to metformin monotherapy on glycemic control in patients with type 2 diabetes. Diabetes Care 1999;22:119-24.

18. Segal P. Efficacy and safety of miglitol therapy com pared with glibenclamide in patients with NIDDM inadequatelly controlled by diet. Diabetes Care 1997: 20:687.

19. Jenmey A, Proietto J, O'Dea K, Nankervis A, Traianedes $K, D^{\prime} E m b d e n$ E. Low-dose acarbose improves glycemic control in NIDDM patients without change in insulin sensitivity, Diabetes Care 1993: 16:499-502.

20. Coniff RF, Shapiro JA, Seaton TB, Bray GA. Multicenter, placebo-controlled trial comparing acarbose (Bay $\mathrm{g}$ 5421) with placebo, tolbutamide, and tolbutamide-plusacarbose in non-insulin-dependent diabetes mellitus. Am J Med 1995:98:443-51

21. Iwamoto $Y$, Kosaka I, Kuzuya T, Akanuma $Y$, Shigeta $Y$, Kaneko T. Effect of combination therapy of troglitazone and sulphonylureas in patients with type 2 diabetes who were poorly controlled by sulphonylurea therapy alone. Diabet Medic 1996;13:365-70 
22. Horton ES, Whitehouse F, Ghazzi MN, et al. Troglitazone in combination with sulfonylurea restores glycemic control in patients with type 2 diabetes. Diabetes Care 1998:21:1462.

23. Day C. Thiazolidinediones: a new class of anti-diabetes drug. Diabet Medic 1999:16:179-92.

24. Balfour JA, Plosker GL. Rosiglitazone. Drugs 1999:57 921-30.

25. Araújo LMB, Ursich MJ, Pupo A, Fukui RT. The effects of glibenclamide treatment on insulin secretion and on insulin binding to erythrocyte in type 2 diabetes mellitus Braz J Med Biol Res 1988;21:35-42.

26. Peters AL, Davidson MB. Insulin plus a sulfonylurea agent for treating type 2 diabetes. Ann Intern Med $1991 ; 115: 45-53$.

27. Kay TB. Triple oral antidiabetic therapy, J Diabetes Compl 1998; 12:311-3.

28. Wolffenbuttle BHR, Landgraf R. A 1 year multicenter randomized double-blind comparison of repaglinide and glyburide for the treatment of type 2 diabetes. Diabetes Care 1999;22:463-7.

29. Lebovitz HE. Insulin secretagogues: old and new. Diabetes $\operatorname{Rev} 1999 ; 7: 139-53$

30. Goldberg RB, Einhorn D, et al. A randomized placebocontrolled trial of repaglinide in the treatment of type 2 diabetes. Diabetes Care 1998;21:1887-903.

31. Scheen AJ. Tratamento pela redução agressiva de peso no manejo do diabetes tipo 2. Diabetes Metab $1998 ; 2: 140$

32. Bray $G$. Drugs used in the treatment of obesity. Diabetes Rev 1997:5:143-63.

33. Weintraub M, Rubin A, Golik A, Byrne L, Scheinbaum ML, Sibutramine in weight control: a dose-ranging, efficacy study. Clin Pharmacol Ther 1991;50:330-7.

34. Hollander PA. Role of orlistat in the treatment of obese patients with type 2 diabetes: a 1-year randomized double study. Diabetes Care 1998:21:1288-94.

35. Berger $M$, Jörgen V. Mühlhauser I. Rationale for use of insulin therapy alone as the pharmacological treatment of type 2 diabetes. Diabeles Care 1999:22(suppl.3):C71-5.

36. Coniff RF, Shapiro JA, Seaton TB, Hoogwerf BJ, Hunt JA. A double-blind placebo controlled trial evaluating the safety and efficacy of acarbose for the treatment of patients with insulin-requiring type 2 diabetes. Diabetes Care 1999; 18:928-32

37. Brunelle RL, Llewelyn J, Anderson JHJr, Gale EAM, Koivisto VA. Meta-analysis of the effect of insulin lispro on severe hypoglycemia in patients with Type 1 diabetes. Diabetes Care 1998:21:1726-31.

38. Bolli GB, Di Marchi RD, Park GD, Pramming S, Koivisto VA Insulin analogues and their potential in the management of diabetes mellitus. Diabetologia 1999:42:1151-67.

39. Kolvisto VA, Tuominem JA, Ebiling P. Lispro MIX25 insulin as premeal therapy in Type 2 diabetes patients. Diabetes Care 1999;22:459-62.

40. Landstedt-Hallin L, Adamson U, Arner P, Bolinder J, Lins PE. Comparison of bedtime NPH or preprandial regular insulin combined with glibenclamide in secondary sulfonylurea failure. Diabetes Care 1995;18:1 183-6.

41. American Diabetes Association. Standards of medical care for patients with diabetes mellitus. Diabetes Care 2000:23(suppl 1):S32-41.

\section{Endereço para correspondência:}

Leila Maria Batista Araújo

Av. Sete de Setembro 2417, ap. 601

40080-003 Salvador, Bahia.

Fax: (071) 247-8492

e-mail: Imba@ufba.br 